\title{
Characteristics of Solar Wind Fluctuations at and below Ion Scales
}

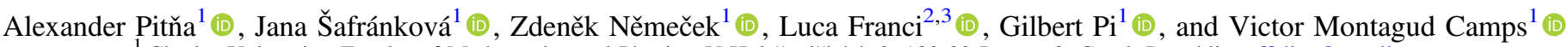 \\ ${ }^{1}$ Charles University, Faculty of Mathematics and Physics, V Holešovičkách 2, 18000 Prague 8, Czech Republic; offelius@ gmail.com \\ School of Physics and Astronomy, Queen Mary University of London, London E1 4NS, UK \\ ${ }^{3}$ INAF, Osservatorio Astrofisico di Arcetri, Largo E. Fermi 5, I-50125 Firenze, Italy \\ Received 2019 February 18; revised 2019 May 6; accepted 2019 May 17; published 2019 July 9
}

\begin{abstract}
Kinetic-scale fluctuations in magnetized collisionless plasmas, such as a solar wind, attract attention owing to their vital role in the dynamics of the dissipation of free energy to random particle motion. As the free energy cascades in the inertial range of turbulence, fluctuations at ion characteristic scales become more compressible. Measurements show that these fluctuations possess highly oblique propagation angles with respect to the background magnetic field and follow theoretical predictions for kinetic Alfvén waves (KAWs). We performed a large (465 cases) statistical study of normalized fluctuations of the density, bulk velocity, and magnetic field around ion gyroscale and concentrated on (i) their compressibility, (ii) the ratio of density and magnetic field fluctuations, and (iii) the ratio of density and velocity fluctuations. We find that observed fluctuations follow the two-fluid prediction for KAWs generally, but the spread of measured values around their theoretical predictions is large. The analysis of measurement uncertainties shows that the difference between the observed and predicted levels of fluctuations cannot be fully explained by these uncertainties and that the nature of solar wind fluctuations is more complex.
\end{abstract}

Key words: solar wind - turbulence

\section{Introduction}

Solar wind, a collisionless magnetized plasma that originates in the solar corona, exhibits fluctuations in magnetic field and plasma moments whose length scales span many orders of magnitude. These fluctuations are turbulent in nature owing to large Reynolds numbers that are characteristic for this supersonic flow. It is believed (Matthaeus et al. 1999; Cranmer et al. 2009; Bruno \& Carbone 2013) that the energy within fluctuations at large scales $\left(\approx 10^{6} \mathrm{~km}\right)$ is transferred via a turbulent cascade into small-scale fluctuations $\left(\approx 10^{2} \mathrm{~km}\right)$, where they are finally damped and their energy is dissipated and heats the solar wind. Interpretation of observations based on the Yaglom/von Kármán-Horwarth equation (Politano \& Pouquet 1998) shows that the energy flux within the inertial range cascade is of the order of dissipation rate that would account for the nonadiabatic proton temperature profile during the solar wind expansion (MacBride et al. 2008; Cranmer et al. 2009; Hellinger et al. 2013). On the other hand, the second-order moments such as power spectral density (PSD) provide more information on the nature of wave modes/structures in the inertial and kinetic ranges.

In the sub-ion range, a number of kinetic effects has been suggested to play either significant or, at least, a partial role in the shaping of kinetic-scale fluctuations and their dynamics, i.e., cyclotron resonance (Coleman 1968; Smith et al. 2012), Landau damping (Howes et al. 2006; Schekochihin et al. 2009), Hall coupling (Galtier 2008; Hellinger et al. 2018; Papini et al. 2019), Alfvén vortexes (Alexandrova et al. 2006; Lion et al. 2016; Perrone et al. 2017), and reconnecting current sheets (Cerri \& Califano 2017; Franci et al. 2018b). How these (and other) effects contribute to the transfer of turbulent energy into particles is still an open question.

The second-order moments, such as PSD, provide more information on the nature of wave modes/structures in the inertial and kinetic ranges. PSDs of the density, $N$, velocity, $\boldsymbol{V}_{\mathrm{sw}}$, and magnetic field, $\boldsymbol{B}$, have been studied in detail by many authors (see reviews, e.g., Tu \& Marsch 1995; Bruno \& Carbone 2013; Chen 2016). In the inertial range, magnetic field fluctuations exhibit $k$-dependent anisotropy (Horbury et al. 2012; Oughton et al. 2015). The first kind of anisotropy is that fluctuations perpendicular to the local mean field, $\delta B_{\perp}$, are much larger than the parallel ones, $\delta B_{\|}$(Belcher \& Davis 1971). The second type of anisotropy is that the total power of the fluctuations depends on the angle $\theta_{B V}$ between the average magnetic field, $\boldsymbol{B}_{0}$, and solar wind speed, $\boldsymbol{V}_{\mathrm{sw}}$ (Horbury et al. 2008; Podesta 2009; Wicks et al. 2010). The theoretical frameworks of weak (Iroshnikov 1963; Kraichnan 1965) or strong (Goldreich \& Sridhar 1995) turbulence phenomenology are used to explain these observations. Osman \& Horbury (2007), Podesta (2009), Chen et al. (2010), and Forman et al. (2011) show that turbulence in the inertial range is mainly composed of modes with wavevectors $k_{\perp} \gg k_{\|}$and with perpendicular, $k_{\perp}^{-5 / 3}$, and parallel, $k_{\|}^{-2}$, scaling favoring the prediction of Goldreich \& Sridhar (1995). Nevertheless, the inertial range also contains compressive fluctuations ( $\mathrm{Tu} \&$ Marsch 1995), which have even stronger anisotropy than the Alfvénic component (Chen et al. 2012a). Klein et al. (2012) found that the compressive component contains up to $10 \%$ of the energy at the inertial range and that it fits the slow wave mode in linearized Vlasov-Maxwell equations.

Both types of anisotropy translate into the kinetic range of turbulence. This range is usually defined by a change of the PSD steepness (relative to the inertial range). For PSDs of bulk $\boldsymbol{V}_{\mathrm{sw}}$ and thermal velocity, $V_{\mathrm{th}}$, the spectral index steepens to values between -2 and -4 (Šafránková et al. 2016), whereas for $N$ the slope first flattens (Unti et al. 1973; Chen et al. 2013b; Šafránková et al. 2015) and then decreases to a value of -2.8 (Alexandrova et al. 2009; Chen et al. 2012b; Šafránková et al. 2015; Riazantseva et al. 2016; Rakhmanova et al. 2018). In the range between the ion and electron scales, magnetic field fluctuations typically exhibit a spectral scaling similar to that of the density, $\delta B_{\perp} \propto f^{-2.8}$ (Chen et al. 2013a), with a significant 
spread around this value. These features are also observed in simulations of kinetic plasma turbulence in both 2D and 3D (Howes et al. 2011; Boldyrev \& Perez 2012; Franci et al. 2015, 2016, 2018b).

In the kinetic range, two electromagnetic wave modes that are most frequently invoked to characterize fluctuations are the kinetic Alfvén wave (KAW) and oblique whistler wave (Boldyrev et al. 2013). The flattening of the density spectrum at $k \rho_{\mathrm{gp}} \approx 1$, where $\rho_{\mathrm{gp}}$ is the proton thermal gyroradius, and the same scaling of density and magnetic field fluctuations point toward a kinetic Alfvén turbulence. This is partly because the KAW mode is a natural continuation of the oblique Alfvén mode toward higher and more perpendicular wavevectors, and partly due to the virtual absence of the fast-mode fluctuations at the inertial range (Howes et al. 2012). In order to distinguish these two modes, Chen et al. (2013a) developed a method based on the dimensionless ratio of relative levels of the density, $\delta N / N_{0}$, and perpendicular magnetic fluctuations, $\delta B_{\perp} / B_{0}$, where $N_{0}$ and $B_{0}$ are the average density and background magnetic field strength over a given interval, respectively. This ratio is then compared to the prediction for KAW that satisfies (1) $k_{\perp} \gg k_{\|}$, (2) $\omega \ll k_{\perp} V_{\text {th,p }}$, and (3) $1 / \rho_{\mathrm{gp}} \ll k_{\perp} \ll 1 / \rho_{\mathrm{ge}}$, where $\omega$ is the angular frequency of the wave, $V_{\mathrm{th}, \mathrm{p}}$ is the average thermal proton speed, and $\rho_{\mathrm{ge}}$ is the electron gyroradius (Boldyrev et al. 2013). Chen et al. (2013a) analyzed ARTEMIS spacecraft measurements of 17 intervals within the pristine solar wind and found that the ratio $R_{n B_{\perp}}=$ $\left(\delta N / N_{0}\right) /\left(\delta B_{\perp} / B_{0}\right)$ matches the KAW prediction well.

Following the Chen et al. (2013a) approach, many studies compared the observed values of various normalized fluctuations with their theoretical predictions for KAWs or oblique whistlers. Concerning theoretical predictions, Schekochihin et al. (2009) and Boldyrev et al. (2013) derived formulae for compressibility of the magnetic field, for normalized density and magnetic field fluctuations in the framework of electron reduced MHD (ERMHD) and kinetic Vlasov-Maxwell equations, respectively. Zhao et al. (2014) studied linear properties of KAWs and kinetic slow waves using two-fluid MHD framework (Hollweg 1999). They derived relations between density and parallel magnetic field fluctuations and for the Alfvén ratio, $R_{\mathrm{A}}\left(R_{\mathrm{A}}\right.$ is the ratio between the kinetic and magnetic energy of fluctuations). Roberts et al. (2018) applied the theoretical formulae of Zhao et al. (2014) for $R_{\mathrm{A}}$ and the Boldyrev et al. (2013) prediction for $R_{n B_{\perp}}$ and found that kinetic range fluctuations measured by the Magnetospheric Multiscale (MMS) mission in Earth's magnetosheath are consistent with kinetic Alfvén-like turbulence. Recently, Grošelj et al. (2018) analyzed high-cadence magnetic field (MMS and Cluster) and plasma measurements $(M M S)$ in the solar wind and reported the agreement between KAW linear predictions of three dimensionless ratios, compressibility, $R_{n B_{\perp}}$, and $R_{n B_{\|}}=\left(\delta N / N_{0}\right) /\left(\delta B_{\|} / B_{0}\right)$, with observations and high-resolution 3D fully kinetic simulations. $\mathrm{Wu}$ et al. (2019) extended the work of Verscharen et al. (2017) into the kinetic range and found that magnetosheath measurements of fluctuations with $k_{\perp} \rho_{\mathrm{gp}} \simeq 2$ fit to KAW-like turbulence (where $\rho_{\mathrm{gp}}=v_{\mathrm{th}} / \omega_{\mathrm{cp}}$ is the ion gyroscale, $v_{\mathrm{th}}$ is the perpendicular thermal speed, $\omega_{\mathrm{cp}}=q_{\mathrm{p}} B_{0} / m_{\mathrm{p}}$ is the proton gyrofrequency, $q_{\mathrm{p}}$ is the proton charge, $m_{\mathrm{p}}$ is the proton mass, and $B_{0}$ is the magnitude of the background magnetic field). They derived relations for four dimensionless ratios of two-fluid MHD together with the expected values of the kinetic Vlasov-Maxwell using the New Hampshire Dispersion relation Solver code (Verscharen \& Chandran 2018).
They argued that the KAW-like fluctuations follow two-fluid predictions and not the kinetic theory. The fact that solar wind fluctuations behave fluid-like confirms also a comparison of observations with numerical solutions for a combination of KAW and slow waves (Šafránková et al. 2019). Finally, Lacombe et al. (2017) analyzed magnetic field fluctuations between characteristic ion and electron kinetic scales and found that the magnetic compressibility of fluctuations in the range of 1 to $15-20 \mathrm{~Hz}$ follows the expected value for KAWs.

In this paper, we present the analysis of three dimensionless quantities, namely, (1) the magnetic compressibility, (2) the ratio of normalized density to normalized perpendicular magnetic fluctuations, and (3) the ratio of normalized density to ion velocity fluctuations. These ratios are estimated from spacecraft measurements and compared with their theoretical predictions for KAWs. The paper is organized as follows. After the introduction (Section 1), the used instruments (Section 2) and the data set, including its discussion and data selection (Section 3), are present. Section 4 provides the definition of analyzed ratios, their theoretical predictions, and relations to the observed levels of fluctuations estimated by continuous wavelet transform (CWT). In Section 5, we compare observed and predicted values for these ratios and present their $k$-dependence. Due to the varying spread of the observed/predicted values, we perform a Monte Carlo (MC) simulation that evaluates a contribution of the measurement errors to the uncertainty of these values (Section 6). The discussion of results and conclusions is in Section 7.

\section{Data Sources}

The Bright Monitor of Solar Wind (BMSW) instrument on board the Spektr-R spacecraft was used for an estimation of ion density fluctuations. Its high sampling rate of $32 \mathrm{~ms}$ provides an excellent opportunity for the analysis of turbulent fluctuations around and below characteristic ion scales (Šafránková et al. 2013b, 2015). It consists of six Farady cups that collect the incoming flux of solar wind ions. We analyzed data acquired in the so-called adaptive mode, where plasma moments are estimated from three points of the velocity distribution function under the assumption of a Maxwellian distribution. The field of view of about $\pm 50^{\circ}$ is centered around the sunward direction; thus, the instrument is insensitive to particles streaming sunward. Electrons are repulsed by $300 \mathrm{~V}$ of the DC potential applied on one Faraday cup grid, and the selection of points for calculations of the moments excludes alpha particles. The design of the instrument does not facilitate a determination of the temperature anisotropy. For further details of computations see Šafránková et al. (2013a). Unfortunately, Spektr-R does not measure the magnetic field; thus, Wind was chosen because it provides a nearly continuous measurement of the magnetic field vector with a sufficient sampling frequency, together with proton and electron plasma moments. The nominal resolution of the Solar Wind Experiment (SWE) measurements is $92 \mathrm{~s}$ for protons and $12 \mathrm{~s}$ for electrons, respectively (Ogilvie et al. 1995). About $0.1 \mathrm{~s}$ resolution magnetic field measurements from the Magnetic Field Investigation (MFI) instrument (Lepping et al. 1995; Koval \& Szabo 2013) are used. 

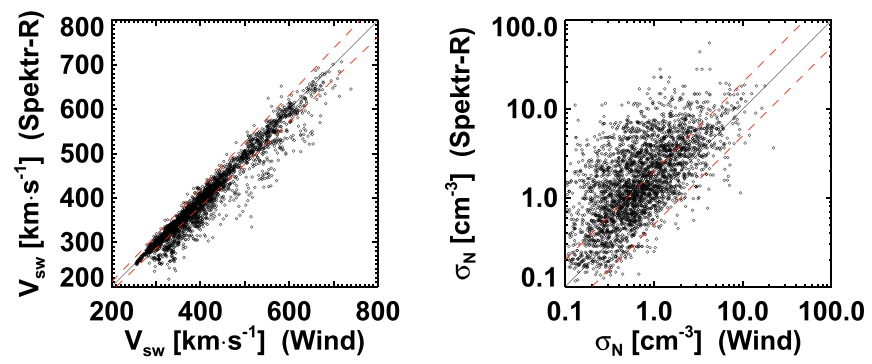

Figure 1. Left panel: comparison of the average solar wind speed measured by SWE (Wind) and BMSW (Spektr-R). The averages were computed over 30minute-long time windows. Right panel: comparison of the standard deviations of ion densities (SWE vs. BMSW) over the same windows.

\section{Data Set}

The time range of analyzed data starts on 2011 September and ends on 2018 May. Our study relies on an assumption that the plasmas observed at Wind and Spektr-R positions have the same properties in a statistical sense. Considering the separation between Wind and Spektr-R along the solar wind flow (roughly the $x$-axis of the GSE coordinate system, $\Delta R$ ), we identify the same solar wind stream for a particular time interval in Wind data using the following procedure: (1) we choose a desired interval, $t_{1}-t_{2}$, at Spektr-R; (2) calculate the mean solar wind speed within this interval, $\left\langle V_{\mathrm{sw}}\right\rangle$; and (3) determine the corresponding interval in the Wind data as $\left(t_{1}-t_{2}\right)-\Delta t$, where $\Delta t=\Delta R /\left\langle V_{\mathrm{sw}}\right\rangle$. A length of the time interval $\left(t_{2}-t_{1}\right)$ was chosen to be 30 minutes. The determination of the appropriate time lag does not ensure that Wind and Spektr-R are in the same solar wind stream. Moreover, the Spektr-R apogee is only $\approx 50 R_{\mathrm{E}}$; thus, it can be sometimes close to the bow shock. For these reasons, we further selected the intervals in accord with the following criteria: (a) the average solar wind speeds, $\left\langle V_{\mathrm{sw}}\right\rangle$, on both spacecraft should satisfy $0.95 \leqslant$ $\left\langle V_{\mathrm{sw}}^{\text {Wind }}\right\rangle /\left\langle V_{\mathrm{sw}}^{\text {Spektr- } R}\right\rangle \leqslant 1.05$; and (b) the standard deviations of ion density measurements estimated over the entire interval (30 minutes), $\sigma_{N}$, satisfy $0.5 \leqslant \sigma_{N}^{\text {Wind }} / \sigma_{N}^{\text {Spektr }-R} \leqslant 2$. Moreover, we used only the observations when Spektr-R was located in the positive $x_{\mathrm{GSE}}$ coordinate. These criteria discard the intervals when Spektr-R is orbiting in the magnetosheath or foreshock. Figure 1 illustrates our selection of intervals for further data processing, which lie between dashed red lines in the figure. A similar procedure was used by Šafránková et al. (2019) for a study of polarization properties of velocity fluctuations. The authors have shown that the mean magnetic field magnitude and direction determined from Wind measurements can be used for a statistical evaluation of Spektr-R plasma measurements.

We estimate the level of fluctuations by computing PSDs of magnetic field components and magnitude (from Wind, MFI) and ion density and solar wind velocity components (Spektr-R, BMSW) via CWT (Torrence \& Compo 1998) for each 30-minute interval. The Morlet mother wavelet with $\omega=6$ was chosen. When the level of magnetic field fluctuations is low, the spectra are often spoiled by a significant noise at higher spacecraft frame frequencies, $f \gtrsim 2 \mathrm{~Hz}$. For this reason, we apply the following interval selections: (1) We estimate the local slope, $\alpha$, of PSD of $|\boldsymbol{B}|$ at the gyrostructure frequency, $f_{\mathrm{gp}}$, because this frequency, defined as $f_{\mathrm{gp}}=V_{\mathrm{sw}} / 2 \pi \rho_{\mathrm{gp}}$, where $V_{\mathrm{sw}}$ is the solar wind bulk speed, has been previously suggested to be related to the spectral break between the MHD and kinetic scales (e.g., Schekochihin et al. 2009). (2) The local slopes at $f_{\mathrm{gp}}$ and at twice this frequency, $\alpha_{f_{\mathrm{gp}}}$ and $\alpha_{2 f_{\mathrm{gp}}}$, respectively, were
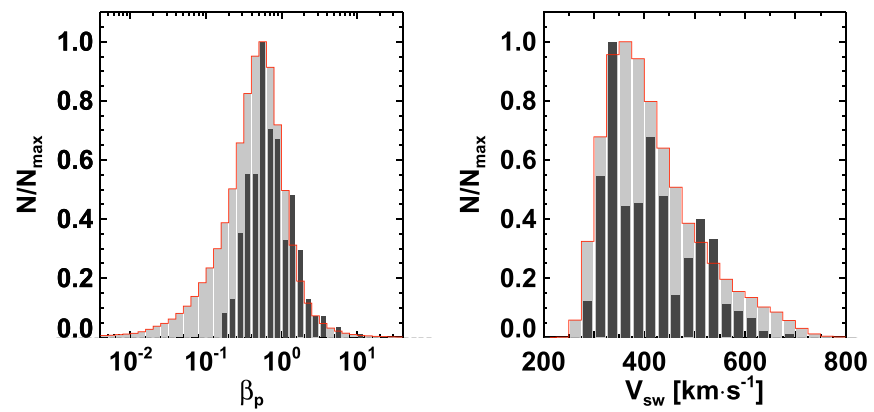

Figure 2. The pdf's of $\log _{10}\left(\beta_{\mathrm{p}}\right)$ (left panel) and $V_{\mathrm{sw}}$ (right panel). Black pdf's mark quantities within our sample, whereas gray pdf's added by the curve mark pdf's estimated from the Wind data set during the time span of our statistics.

used to remove the intervals for which $\alpha_{f_{\mathrm{gp}}}<-2$ or $\alpha_{2 f_{\mathrm{gp}}}<-2$. (3) Since we are interested in the transition from inertial to kinetic ranges, our frequency range should cover this transition. We use the third constraint, ensuring that the spectral break lies within our range, thus $f_{\mathrm{gp}} \leqslant 2 \mathrm{~Hz}$. The final number of intervals remaining for further analysis is 465 .

We would like to apply our analysis on all solar wind measurements, but our selection of samples is not random. In order to quantify applicability of our set after selections, Figure 2 shows the histograms of the mean proton beta, $\beta_{\mathrm{p}}=\left(2 \mu_{0} k_{\mathrm{B}} N T_{\mathrm{p}}\right) / B^{2}$, and mean solar wind speed, $V_{\mathrm{sw}}$, corresponding to selected intervals derived from the Wind data, where $\mu_{0}$ is the permeability of vacuum, $k_{\mathrm{B}}$ is the Boltzmann constant, and $T_{\mathrm{p}}$ is the proton temperature. A number of events in bins is normalized to the maximum in each particular set. One can see that $\beta_{\mathrm{p}}$ varies between 0.094 and 14.5, whereas $V_{\mathrm{sw}}$ lies between 282 and $679 \mathrm{~km} \mathrm{~s}^{-1}$. Light-gray histograms in both panels of Figure 2 represent the background distribution of the corresponding quantities for 2011-2017. For solar wind speed distributions, the two-sample KolmogorovSmirnov (K-S) statistical test yields the significance level of 0.052 , i.e., the null hypothesis that two samples come from the same distribution cannot be rejected. However, the K-S test of the $\beta_{\mathrm{p}}$ distribution gives the significance level of $10^{-24}$. Figure 2 shows that our statistics is biased toward medium and higher values of $\beta_{\mathrm{p}}$. It is a consequence of our selection criteria, mainly due to the limitation $f_{\mathrm{gp}} \leqslant 2 \mathrm{~Hz}$. Since $f_{\mathrm{gp}}$ increases with decreasing proton gyroradius, this condition discards intervals with high magnetic fields and low temperatures. This means that the results and conclusions presented in the following sections could be applied on fluctuations observed in environments with $\beta_{\mathrm{p}} \approx 1$.

\section{KAW Normalization}

The proton thermal gyroradius, $\rho_{\mathrm{gp}}$, is the characteristic scale at which KAWs become dispersive (Hasegawa \& Sato 1989; Stix 1992). Therefore, we analyze the fluctuations of $\boldsymbol{B}, N$, and $\boldsymbol{V}_{\mathrm{sw}}$ at $f=f_{\mathrm{gp}}$ and for $f>f_{\mathrm{gp}}$. We compute four nondimensional ratios that characterize the nature of fluctuations: the ratio of the normalized density and perpendicular magnetic fluctuations, $R_{n B_{\perp}}$, magnetic compressibility, $C_{\|}$, the ratio between kinetic and magnetic energies, $R_{\mathrm{A}}$, and the ratio of normalized density and velocity fluctuations, $R_{n V}$. Note here that in calculations of all derived quantities, the Wind measurements are used, except $\delta N / N_{0}$ and $\delta V_{\mathrm{sw}}$, which are taken from Spektr-R. 
1. The ratio $R_{n B_{\perp}}$ is defined as

$$
R_{n B_{\perp}} \equiv \frac{\left(\delta N / N_{0}\right)^{2}}{\left(\delta B_{\perp} / B_{0}\right)^{2}},
$$

where $\delta N^{2}$ is taken as $\operatorname{PSD}(N)$ and $\delta B_{\perp}$ is approximated as $\sum_{i=0}^{2} \operatorname{PSD}\left(B_{i}\right)-\operatorname{PSD}(|\boldsymbol{B}|)$. We note that it assumes parallel fluctuations, $\delta B_{\|}$being close to those of the magnetic field strength $\delta|\boldsymbol{B}|$. At high spacecraft frame frequencies, this approximation is often used (e.g., Chen \& Boldyrev 2017).

2. Magnetic compressibility:

$$
C_{\|} \equiv \frac{\delta B_{\|}^{2}}{\delta B^{2}}
$$

where $\delta B_{\|}^{2} \simeq \operatorname{PSD}(|\boldsymbol{B}|)$ and $\delta B^{2} \simeq \sum_{i=0}^{2} P S D\left(B_{i}\right)$.

3. The ratio $R_{\mathrm{A}}$ :

$$
R_{\mathrm{A}} \equiv \mu_{0} N_{0} m_{\mathrm{p}} \frac{\delta V_{\mathrm{sw}}^{2}}{\delta B^{2}},
$$

where $m_{\mathrm{p}}$ is the proton mass and $\delta V_{\mathrm{sw}}^{2} \simeq \sum_{i=0}^{2} \operatorname{PSD}\left(V_{\mathrm{sw}, i}\right)$.

4. The ratio $R_{n V}$ can be expressed in terms of the ratios defined by Equations (1)-(3):

$$
R_{n V} \equiv \frac{\left(\delta N / N_{0}\right)^{2}}{\left(\delta V_{\mathrm{sw}} / V_{\mathrm{A}}\right)^{2}}=\left(1-C_{\|}\right) \frac{R_{n B_{-}}}{R_{\mathrm{A}}},
$$

where $V_{\mathrm{A}}$ is the Alfvén speed computed from the Wind data.

A theoretical prediction of values of these quantities for KAWs can be calculated using the kinetic Vlasov equations (Boldyrev et al. 2013), or in gyrokinetic (Howes et al. 2006) or fluid (Hollweg 1999) frameworks.

1. We follow Boldyrev et al. (2013) for the prediction of $R_{n B_{\perp}}$ :

$$
R_{n B_{\perp}}^{\mathrm{KAW}}=\frac{1}{K^{2}}=\frac{1}{\frac{\beta_{\mathrm{p}} \mathcal{T}}{2}\left(1+\frac{\beta_{\mathrm{p}} \mathcal{T}}{2}\right)},
$$

where $\mathcal{T}=\left(1+T_{\mathrm{e}} / T_{\mathrm{p}}\right)$.

2. For the KAW compressibility, Boldyrev et al. (2013) derive

$$
C_{\|}^{\mathrm{KAW}}=\frac{\beta_{\mathrm{p}} \mathcal{T}}{2\left[1+\beta_{\mathrm{p}} \mathcal{T}\right]} .
$$

3. The Alfvén ratio for the KAWs was calculated by Zhao et al. (2014), and we adopt a simplified formula that is valid for $\beta_{\mathrm{p}} \lesssim 1$ :

$$
R_{\mathrm{A}}^{\mathrm{KAW}}=\frac{\frac{1}{\Lambda}+\frac{\beta_{\mathrm{p}}}{\rho_{\mathrm{gp}}^{2} k_{\perp}^{2}}\left(\frac{1-\Lambda}{\Lambda}\right)^{2}}{1+\frac{\beta_{\mathrm{p}}}{\rho_{\mathrm{gp}}^{2} k_{\perp}^{2}} \frac{(\Lambda-1)^{2}}{\Lambda}},
$$

where

$$
\begin{aligned}
\Lambda= & \frac{1}{2} \frac{1+2 \mathcal{T} \beta_{\mathrm{p}}+\mathcal{T} \rho_{\mathrm{gp}}^{2} k_{\perp}^{2}}{1+\mathcal{T} \beta_{\mathrm{p}}} \\
& \times\left[1+\sqrt{1-4 \beta_{\mathrm{p}} \frac{1+\mathcal{T} \beta_{\mathrm{p}}}{\left(1+2 \mathcal{T} \beta_{\mathrm{p}}+\mathcal{T} \rho_{\mathrm{gp}}^{2} k_{\perp}^{2}\right)^{2}}} .\right.
\end{aligned}
$$

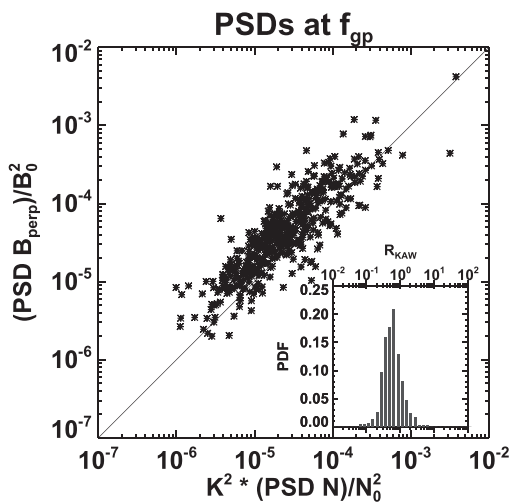

Figure 3. Scatter plot of normalized perpendicular magnetic field fluctuations and KAW normalized density fluctuations at $f=f_{\mathrm{gp}}$ within our data set. $K$ was estimated by Equation (5). The embedded histogram shows the pdf of $R_{\mathrm{KAW}}$ (Equation (9)).

For $\beta_{\mathrm{p}} \ll 1$, this expression reduces to that introduced by Roberts et al. (2018).

4. The ratio of the normalized density and velocity fluctuations can be expressed in accord with Equation (4):

$$
R_{n V}^{\mathrm{KAW}} \equiv\left(1-C_{\|}^{\mathrm{KAW}}\right) \frac{R_{n B_{\perp}}^{\mathrm{KAW}}}{R_{\mathrm{A}}^{\mathrm{KAW}}} .
$$

Finally, we determine ratios of the observed values of $R_{n B_{1}}$, $C_{\|}$, and $R_{n V}$ to their KAW theoretical predictions, $R_{\mathrm{KAW}}$, $C_{\mathrm{KAW}}$, and $A_{\mathrm{KAW}}$, respectively:

$$
\begin{aligned}
R_{\mathrm{KAW}} & =\frac{R_{n B_{\perp}}(\mathrm{obs})}{R_{n B_{\perp}}^{\mathrm{KAW}}}, \\
C_{\mathrm{KAW}} & =\frac{C_{\|}(\mathrm{obs})}{C_{\|}^{\mathrm{KAW}}},
\end{aligned}
$$

and

$$
A_{\mathrm{KAW}}=\frac{R_{n V}(\mathrm{obs})}{R_{n V}^{\mathrm{KAW}}} .
$$

Note that the $R_{\mathrm{KAW}}$ ratio was discussed also in studies of Chen et al. (2013a) and Roberts et al. (2018).

\section{KAWs-Observations versus Theory}

Figure 3, motivated by the Chen et al. (2013a) approach, presents a scatter plot of $K^{2} \delta N^{2} / N_{0}^{2}$ and $\delta B_{\perp}^{2} / B_{0}^{2}$ estimated at $k \rho_{\mathrm{gp}}=1$, where $K^{2}=1 / R_{n B_{1}}$ (Equation (5)). The figure demonstrates that $K^{2}$ can be considered as a normalization factor for the density fluctuations (Chen et al. 2013a). $K^{2}$ is estimated from the Wind measurements of $T_{\mathrm{e}}, T_{\mathrm{p}}, N_{0}$, and $\boldsymbol{B}$, and Figure 3 shows a strong correlation $($ corr $=0.82)$ between both quantities. The diagonal line represents the theoretical prediction for KAWs, and the embedded histogram shows the probability distribution function (pdf) of $R_{\mathrm{KAW}}$ values (Equation (9)) depicted in the figure. The distribution is similar to that shown in Figure 2(a) of Chen et al. (2013a); however, we observe a slightly lower median value, $0.55_{-0.01}^{+0.03}$.

In the following subsections, we compare $R_{n B_{1}}, C_{\|}$, and $R_{n V}$ estimated from the theory and observations and discuss $R_{\mathrm{KAW}}$, $C_{\mathrm{KAW}}$, and $A_{\mathrm{KAW}}$ in more detail. For each quantity, we focus 

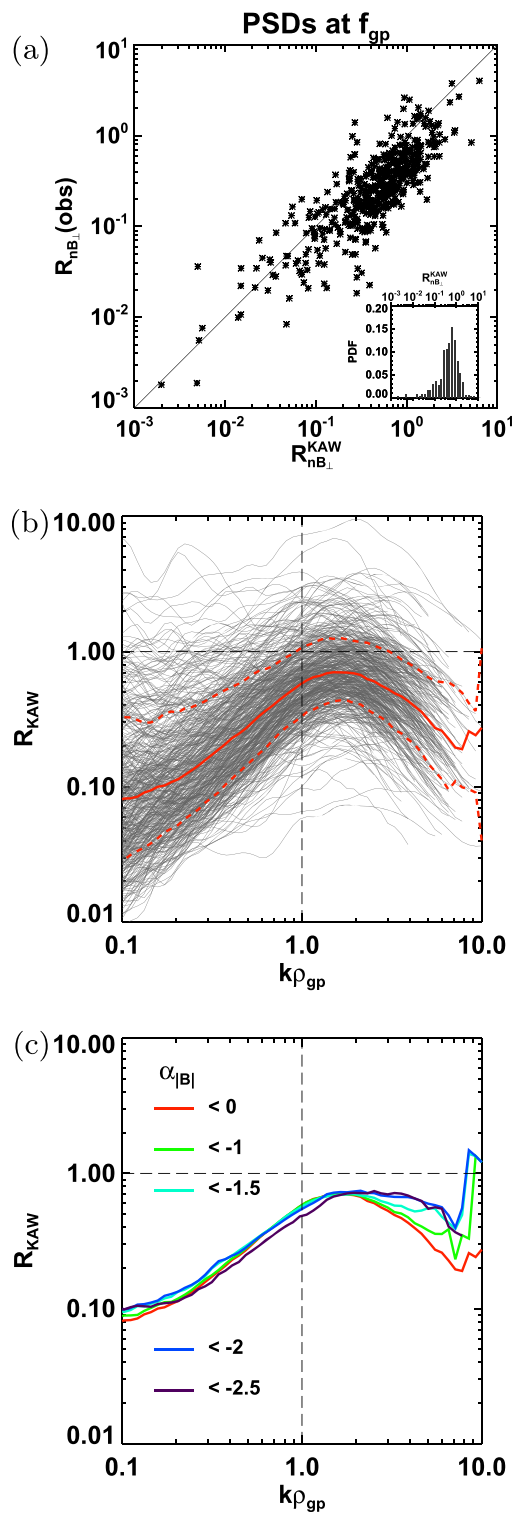

Figure 4. Analysis of $R_{n B_{\perp}}$. (a) Scatter plot of observed and predicted values of $R_{n B_{\perp}}$. The embedded histogram shows the pdf of the predicted value. (b) $R_{\mathrm{KAW}}$ as a function of $k \rho_{\mathrm{gp}}$ for each 30-minute interval (gray curves). The solid red curve denotes the median value of the whole set. Dashed red lines mark the central confidence interval that contains $68 \%$ of values at a given frequency. (c) Colored curves show profiles of medians in different subsets differing by $\alpha_{|\boldsymbol{B}|}$ as given in the panel. Note that the red curves in panels (b) and (c) are identical and belong to the full set.

on its value at the proton gyroscale, $k \rho_{\mathrm{gp}}=1$, and then we show the variations of the values for $k \rho_{\mathrm{gp}}>1$.

\subsection{Density Normalization, $R_{n B_{\perp}}$}

A scatter plot of observed and predicted values of $R_{n B_{\perp}}$ in Figure 4(a) clearly shows that Equation (5) predicts $R_{n B_{\perp}}$ over a broad range of $\beta_{\mathrm{p}}$ (see Figure 2 ) and $\beta_{\mathrm{e}}\left(0.12 \leqslant \beta_{\mathrm{e}} \leqslant 33\right)$ rather well, but the distribution of $R_{n B_{\perp}}^{\mathrm{KAW}}$ in the embedded histogram is non-Gaussian and spans roughly over three orders of magnitude.

On the other hand, the approximation in Equation (5) is not necessarily accurate for $k \rho_{\mathrm{gp}}=1$, but it would be valid for $k \rho_{\mathrm{gp}}>1$. Therefore, we estimated $R_{\mathrm{KAW}}$ for $0.1 \leqslant k \rho_{\mathrm{gp}} \lesssim 10$. The profiles are shown in Figure $4(\mathrm{~b})$ by thin gray lines. The median of the $R_{\mathrm{KAW}}$ profiles is plotted by the solid red line, and the dashed lines denote the 16th and 84th percentiles. A deeper analysis revealed that a part of $R_{\mathrm{KAw}}$ profiles can be spoiled by the noise and aliasing in the magnetic field data, although such intervals were mostly discarded by the algorithm described in Section 3. In order to account for a possible bias above $f_{\mathrm{gp}}$, we estimate a local slope, $\alpha_{|\boldsymbol{B}|}$, of PSD $|\boldsymbol{B}|$ in the frequency range of $2<f<4 \mathrm{~Hz}$. Then, we sorted the cases according to $\alpha_{|\boldsymbol{B}|}$ as follows: the solid red curve in Figure 4(c) marks the median of $R_{\mathrm{KAW}}(f)$ for the whole set, and other colors stand for intervals that gradually satisfy the condition $\alpha_{|\boldsymbol{B}|}<\{-1,-1.5,-2,-2.5\}$. As can be seen in the figure, $R_{\mathrm{KAW}}$ tends to saturate at $\approx 0.7$ for $k \rho_{\mathrm{gp}} \gtrsim 2$ if $\alpha_{|\boldsymbol{B}|}<2$.

\subsection{Compressibility, $\mathrm{C}_{\|}$}

Compressibility of solar wind fluctuations was estimated using Equation (2), whereas theoretically predicted values for KAWs are computed by Equation (6). Note that this quantity is determined with a higher precision than $R_{\mathrm{KAW}}$ discussed in the previous section because all parameters come from one $s / c$ (Wind).

Figure 5(a) shows a scatter plot of observed and predicted values of $C_{\|}$at $k \rho_{\mathrm{gp}}=1$. The result is consistent with the finding of Lacombe et al. (2017) (see their Figure 11). The observed level of compressibility is lower than the KAW prediction, but observations and predictions become closer together $\left(C_{\mathrm{KAW}} \approx 0.9\right.$; see Figure $\left.5(\mathrm{~b})\right)$ for $k \rho_{\mathrm{gp}} \gtrsim 2$.

Although $C_{\mathrm{KAw}}$ of individual intervals differs by two orders of magnitude for $k \rho_{\mathrm{gp}} \approx 0.1$, the profiles tend to converge for large $k \rho_{\mathrm{gp}}$ and the median becomes close to unity for $k \rho_{\mathrm{gp}} \gtrsim 2$ (Figure 5(b)). Figure 5(c) analyzes the influence of the instrumental noise analogously to Figure 4(c). Note that all curves in Figure 5(c) are coming together, unlike the curves in Figure 4(c). In the previous subsection, we have shown that for higher frequencies only the intervals with steepest slopes in the kinetic range are credible (blue lines in Figure 4(c)). Therefore, it is surprising that for $C_{\mathrm{KAW}}$ the lines converge, but the explanation is straightforward. The mean value of the observed compressibility for intervals where instrumental effects are negligible $\left(\alpha_{|\boldsymbol{B}|}<2\right)$ is roughly 0.3 , and the expected value of $C_{\|}$for fluctuations dominated by a white noise is also close to 0.3 .

\subsection{Ratio of Normalized Density and Velocity Fluctuations, $\mathrm{R}_{\mathrm{nV}}$}

The reason for estimation of this ratio rather than the Alfvén ratio (Equation (3)) is that the density and velocity are measured by the same instrument and only the average quantities used in calculations of theoretical and observed values are propagated from Wind. The scatter plot of observed versus predicted $R_{n V}$ in Figure 6(a) reveals their good matching for $f=f_{\text {gp }}$ over three orders of magnitude. Figure 6(b) shows a superposed frequencydependent ratio, $A_{\mathrm{KAW}}$ (Equation (11)). At $f_{\mathrm{gp}}$, the median value of this ratio is lower than unity, whereas for the frequencies above $f_{\mathrm{gp}}, A_{\mathrm{KAw}}$ rises slightly above unity, suggesting that the normalized density and velocity fluctuations roughly follow KAW relations.

\section{Probability Density Functions}

In this section, we focus on pdf's of $R_{\mathrm{KAW}}, C_{\mathrm{KAW}}$, and $A_{\mathrm{KAW}}$. We address the following questions: (i) what is 
(a)
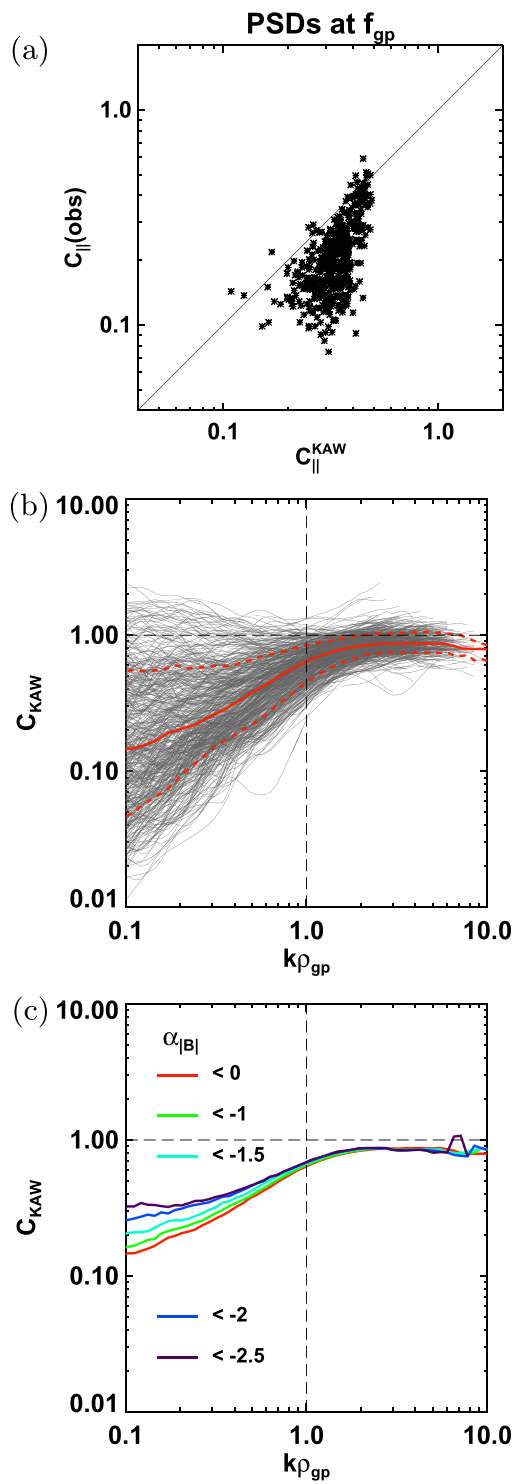

Figure 5. Analysis of $C_{\|}$. (a) Scatter plot of observed and KAW predicted values of $C_{\|}$. (b) $C_{\mathrm{KAW}}$ as a function of $k \rho_{\mathrm{gp}}$ for each 30-minute interval within our set (gray curves). Solid and dashed red curves denote the median and central confidence interval, respectively. (c) Colored curves have the same meaning as in Figure 4(c).

the contribution of uncertainties of the measurements to pdf's, and (ii) how large is the contribution of the errors that are introduced by the propagation from Wind to Spektr-R.

\subsection{Measurement Uncertainty}

As was shown by Chen et al. (2013a), the pdf of $R_{\mathrm{KAw}}$ spans roughly one and a half orders of magnitude. The authors noted that uncertainties of measured quantities contribute to the resulting pdf of $R_{\mathrm{KAW}}$. However, they did not show how large this contribution is or whether it can explain the width of the pdf.

To characterize the width of the distributions for our set of intervals, we calculated corresponding variances as $\mathrm{var}=$ $\left\langle Q_{\mathrm{KAW}}^{2}\right\rangle-\left\langle Q_{\mathrm{KAW}}\right\rangle^{2}$, where $Q \in\left\{R_{\mathrm{KAW}}, C_{\mathrm{KAW}}, A_{\mathrm{KAW}}\right\}$. Since variances are functions of frequency, they were calculated for $f=2 f_{\mathrm{gp}}$, because the median values are almost saturated around
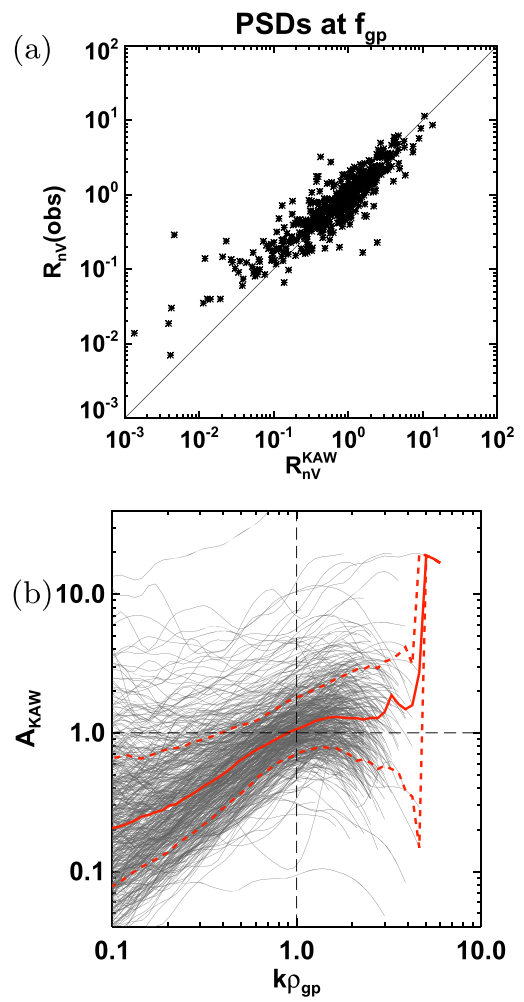

Figure 6. Analysis of $A_{\mathrm{KAW}}$. (a) Scatter plot of observed and KAW predicted values of $R_{n V}$ at $f=f_{\mathrm{gp}}$. (b) $A_{\mathrm{KAW}}$ as a function of $k \rho_{\mathrm{gp}}$ for each 30-minute interval (gray curves). Solid and dashed red curves have the same meaning as in Figures 4(c) and 5(c).
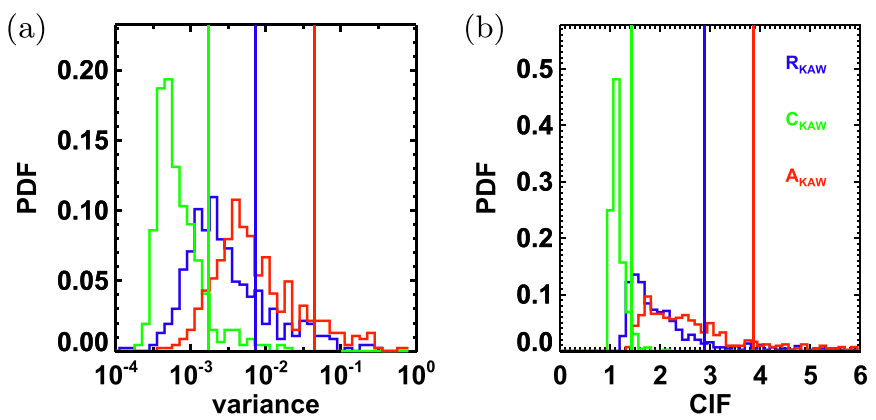

Figure 7. Results of MC simulations of uncertainties of $R_{\mathrm{KAW}}$ (green), $C_{\mathrm{KAW}}$ (blue), and $A_{\mathrm{KAW}}$ (red) at $k \rho_{\mathrm{gp}}=2$. (a) The pdf's of the variances. Each simulation has $10^{5}$ runs for each interval and each quantity. (b) The pdf's of the CIFs. The colored vertical lines mark the variances and CIFs estimated from the original set.

this frequency (see Figures 4(c), 5(c), and 6). The variances are shown by the vertical lines in Figure 7(a).

The second quantity used for a characterization of the pdf's of $R_{\mathrm{KAW}}, C_{\mathrm{KAW}}$, and $A_{\mathrm{KAW}}$ is the confidence interval factor (CIF). Provided that the generally used central confidence interval (CI) is bounded by $\left[c i_{\text {lower }}, c i_{\text {upper }}\right]$, CIF is defined as $\mathrm{CIF}=c i_{\text {upper }} / c i_{\text {lower }}$. The values of CIF for our intervals are depicted as vertical lines in Figure 7(b). Note that CIF has a similar meaning to the relative standard deviation, but we think that CIF is more robust for a description of highly nonGaussian pdf's because it is less sensitive to outliers. The difference between these two quantities is analogous to the difference between mean and median values.

Our estimation of the influence of measuring uncertainties and noise on resulting values of $R_{\mathrm{KAW}}, C_{\mathrm{KAW}}$, and $A_{\mathrm{KAW}}$ is 
based on MC simulations. For each of our intervals, we used the mean values of the magnetic field, velocity, and temperatures and their standard deviations. Assuming a normal distribution of measuring errors of each quantity with the width given by the standard deviation computed on the corresponding interval, we determined $10^{5}$ simulated values of $R_{\mathrm{KAW}}, C_{\mathrm{KAW}}$, and $A_{\mathrm{KAW}}$ using Equations (1), (2), and (4). A detailed description of the simulation procedure can be found in the Appendix.

The results of MC simulations are shown in Figure 7 as colored histograms and can be summarized as follows. The variance of the distribution of $R_{\mathrm{KAW}}$ is larger than $\approx 80 \%-90 \%$ of the variances of the simulated error distributions, and the same holds for $C_{\mathrm{KAW}}$ and $A_{\mathrm{KAw}}$. If the uncertainties of measurements were the only errors contributing to the variance of pdf's of $R_{\mathrm{KAW}}$, one would expect this percentage to be $\approx 50 \%$. Moreover, mean values of simulated variances are approximately one order of magnitude lower than the observed values. This suggests that the measurement uncertainties contribute only slightly to a width of the distributions of observed KAW ratios. Figure 7(b) shows the distribution of CIFs of simulated error distributions, and the conclusion is the same as for the variances. CIF for $C_{\mathrm{KAW}}$ is around 1.5, whereas CIFs of $R_{\mathrm{KAW}}$ and $A_{\mathrm{KAW}}$ are $\approx 2.9$ and $\approx 3.8$, respectively. The distributions of CIFs from MC simulations are well below the CIFs of each quantity, in agreement with the previous Figure 7(a). Generally, the figure confirms our suggestion that the uncertainties of measurements are not able to explain the width of pdf's of analyzed ratios.

\subsection{Propagation Uncertainty}

We argue that the uncertainty introduced by the propagation $($ Wind $\rightarrow$ Spektr-R) also cannot explain the width of the distributions. The key point of the argument is based on a comparison of the distribution of $R_{\mathrm{KAW}}$ obtained by Chen et al. (2013a) with that from our statistical analysis. Assuming that the uncertainties entering their calculations are similar to ours, and taking into account the results of the previous subsection, we may state that the pdf's that they observe are not significantly influenced by the measurement uncertainties. Since their and our distributions of $R_{\mathrm{KAW}}$ cover a similar range of $R_{\mathrm{KAW}} \in[\approx 0.1, \approx 5]$, we can conclude that the propagation error is small.

Furthermore, values of PSDs that enter the $R_{n V}$ computation are from Spektr-R, and only averaged $v_{A}$ from Wind is used, whereas $R_{n B_{\perp}}$ requires $\delta B_{\perp}$ (Wind) and $\delta N($ Spektr-R); thus, the influence of a propagation should be stronger for this quantity. However, Figure 7(b) shows that CIF for $R_{\mathrm{KAW}}$ is even smaller than that of $A_{\mathrm{KAw}}$. This fact also confirms the suggestion that the uncertainty introduced by the propagation is small.

\section{Discussion and Conclusion}

We performed a statistical study of fluctuations at and below the characteristic scale of the proton thermal gyroradius. We analyzed three derived physical quantities that can characterize the nature of fluctuations: (i) the dimensionless ratio of normalized fluctuations of density and perpendicular magnetic field fluctuations, $\left(\delta N / N_{0}\right) /\left(\delta B_{\perp} / B_{0}\right)$, where we combined measurements from Wind and Spektr-R; (ii) compressibility of magnetic field fluctuations, $\delta B_{\|} / B_{0}$, which was estimated solely from Wind measurements; and (iii) the ratio of normalized density and ion bulk velocity fluctuations, $\left(\delta N / N_{0}\right) /\left(\delta V_{\mathrm{sw}} / V_{\mathrm{A}}\right)$, estimated from Spektr-R measurements except $V_{\mathrm{A}}$. These three experimental ratios were compared with two-fluid MHD and/ or kinetic predictions for KAWs. We defined three frequencydependent ratios of these observed/predicted values and analyzed them in detail. Below, we discuss the results of our analysis.

Median values of $R_{\mathrm{KAW}} \approx 0.7, C_{\mathrm{KAW}} \approx 0.9$, and $A_{\mathrm{KAW}} \approx 1.2$ at $k \rho_{\mathrm{gp}}=2$ (Figures 4(a), 5(a), 6(a)) show that the observed fluctuations of $\delta V_{\mathrm{sw}}, \delta B$, and $\delta N$ roughly follow the relations of linear KAWs with $k_{\perp} \gg k_{\|}$. However, the distributions of these ratios are strikingly different. For the compressibility, the factor that estimates a deviation of the observation from the theory, CIF, is roughly 1.4 (Figure 7(b)). In the comparison, CIFs for $R_{\mathrm{KAW}}$ and $A_{\mathrm{KAW}}$ are $\approx 2.9$ and $\approx 3.8$, respectively. MC simulations of the errors of these ratios suggest that such a spread cannot be explained by the uncertainties of measurements.

We continue the discussion of uncertainties because we believe that it should be taken into account in studies of solar wind turbulence. It concerns systematic errors in estimations of basic quantities such as density and solar wind speeds (thermal, electron, proton, etc.). In a majority of studies, the analyzed data come from one spacecraft only; therefore, it is usually not addressed. However, in our study we combine measurements from two spacecraft; thus, it is necessary to compare the proton density estimated from SWE $\left(N_{\text {Wind }}^{\mathrm{SWE}}\right)$ and BMSW $\left(N_{\text {Spektr-R }}^{\mathrm{BMSW}}\right)$ instruments. We found $\left\langle N_{\text {Wind }}^{\text {SWE }} / N_{\text {Spektr-R }}^{\text {BMSW }}\right\rangle \sim 0.85_{-0.12}^{+0.11}$. For the comparison, we estimated average density $N_{\mathrm{ACE}}^{\mathrm{SWE}}$ from the SWEPAM instrument on board the $A C E$ spacecraft within intervals corresponding to our set and found $\left\langle N_{\text {Wind }}^{\mathrm{SWE}} / N_{\mathrm{ACE}}^{\mathrm{SWE}}\right\rangle \sim 1.24_{-0.21}^{+0.15}$. These offsets are not caused by different spacecraft positions but result from a combination of systematic errors of particular instruments and data processing methods. For example, both Wind SWE and Spektr-R BMSW use Faraday cups for the density determination. However, BMSW in the adaptive mode provides a total ion current, and the density is estimated under the assumption that all ions are protons, whereas SWE measures a 3D ion velocity distribution that is fitted with two bi-Maxwellian distributions corresponding to protons and alpha particles. Neglecting the alpha contribution in BMSW thus can lead to an overestimation of the proton density by a factor of 1.03-1.15. On the other hand, ACE SWEPAM uses the top head electrostatic analyzer and channeltrons for measurements of a 3D velocity distribution. Since a registration efficiency of these detectors changes in time, the data are periodically recalibrated by the instrument team.

Because CIF for the pdf of $N_{\text {Wind }}^{\mathrm{SWE}} / N_{\text {Spektr-R }}^{\mathrm{BMSW}}$ is roughly 1.3 , the contribution of this uncertainty to the width of pdf's of $R_{\mathrm{KAW}}$ or $A_{\mathrm{KAW}}$ is small, whereas their median values are affected. It is reasonable to assume that this error is less than a factor of 2. For example, measurements of compressibility (see Figures 5(b) and 7) suggest that errors in proton and electron temperatures are well within a factor of 1.5 .

It is likely that a temperature anisotropy, $T_{\perp} / T_{\|}$, could alter the theoretical prediction of $R_{n B_{\perp}}^{\mathrm{KAW}}, C_{\|}^{\mathrm{KAW}}$, and $R_{n V}^{\mathrm{KAW}}$ for various $\beta_{\mathrm{p}}$. Numerical simulations performed with the hybrid particle-in-cell (HPIC) code CAMELIA (Franci et al. 2018a) suggest that a level of temperature anisotropy influences the values of $R_{n B_{\perp}}$ and $R_{n V}$ (L. Franci et al. 2019, in preparation). We plan to address the effect of anisotropy in a future study.

An interplay between the complex shape of the velocity PSDs around the ion spectral break and the overestimated spacecraft 
frame frequency $f_{\mathrm{gp}}$ introduces another uncertainty. Following the Taylor hypothesis, $\omega_{\mathrm{sc}} \simeq \boldsymbol{k} \cdot \boldsymbol{V}_{\mathrm{sw}} \simeq k_{\perp} V_{\mathrm{sw}} \sin \theta_{B V}$, where $\theta_{B V}$ is the angle between the mean magnetic field and solar wind flow, we set $\theta_{B V}=90^{\circ}$ in the analysis. However, $\theta_{B V}$ in our statistics covers the range $\left[0^{\circ}, 90^{\circ}\right]$ nonuniformly with a median value of $\approx 60^{\circ}$. From this it follows that spacecraft frame frequencies at which the PSDs were analyzed are on average by a factor of two higher than those correctly estimated from the Taylor hypothesis. Ratios of the PSD values at these frequencies will be slightly different from those we analyzed.

Finally, the median frequency profiles of $R_{\mathrm{KAW}}$ and $C_{\mathrm{KAW}}$ are close to those estimated by Franci et al. (2018b) that ran large-size hybrid-kinetic 3D simulations of freely decaying turbulence with $\beta_{\mathrm{p}}=\beta_{\mathrm{e}}=0.5$. For the same plasma $\beta$, Grošelj et al. (2018) used fully 3D kinetic simulations and obtained similar profiles around the proton gyroscale. Our measurements show that although kinetic-scale fluctuations globally follow KAW predictions, there is a significant spread in the estimated $R_{\mathrm{KAW}}$ and $A_{\mathrm{KAW}}$ that is not caused by measuring errors. We plan to investigate this topic in the following paper, which will employ hybrid-kinetic 3D simulations.

As a conclusion, we summarize that the observed levels of solar wind fluctuations around the ion characteristic scale generally follow theoretical linear relations for KAWs. Our results are consistent with the previous studies (Chen et al. 2013a; Lacombe et al. 2017; Grošelj et al. 2018; Roberts et al. 2018). Moreover, we demonstrate that a spread of the observed/predicted values of KAW normalized fluctuations is not caused by either random or systematic errors of measurements. It means that the nature of the fluctuations is more complex, and we plan to address the largest deviations from a KAW-like behavior in further investigations.

The authors acknowledge the use of the Wind MFI magnetic field and SWE proton data. This work is supported by the Czech Grant Agency under contracts 19-18993S. A.P. thanks the Charles University Grant Agency, project No. 1484217. We appreciate the fruitful discussions with Owen Roberts, Simone Landi, Peter Hellinger, and Honghong Wu. This research was partially supported by the UK Science and Technology Facilities Council (STFC) grant ST/P000622/.

Facilities: Wind, Spektr-R.

\section{Appendix}

We illustrate the MC simulation technique on $R_{\mathrm{KAW}}$, and the same procedure was used for $C_{\mathrm{KAW}}$ and $A_{\mathrm{KAW}}$. Our task is to estimate the error distribution of $R_{\mathrm{KAW}}$ for each interval. Let's denote a set of the estimated ratios of $R_{\mathrm{KAW}}$ in our statistics as $R_{i}$, $i=0, \ldots, N$, where $N=465$ is our number of intervals. Then, $R_{i}=g\left(\boldsymbol{X}_{i}\right)$, where the function $g$ is defined by Equations (1), (5), and (9), and $\boldsymbol{X}_{i}=\left\{N_{\mathrm{p}}, B_{0}, T_{\mathrm{e}}, T_{\mathrm{p}}, \text { PSD } B_{i}\left(2 f_{\mathrm{gp}}\right), \operatorname{PSD}|\boldsymbol{B}|\left(2 f_{\mathrm{gp}}\right)\right\}_{i}$ are the average quantities that enter the calculation of $R_{i}$. For each interval $(i)$, we generate $M=10^{5}$ pseudo-random sets from a multivariate normal distribution with diagonal covariance matrix and corresponding mean values and variances, $\boldsymbol{X}_{i}^{j}=\mathcal{N}^{j}\left(\boldsymbol{X}_{i}, \boldsymbol{\Sigma}_{i}\right)$, where $j=0, \ldots, M$ and $\boldsymbol{\Sigma}_{i}$ stands for either the standard deviations of $\left\{N_{\mathrm{p}}, B_{0}, T_{\mathrm{e}}, T_{\mathrm{p}}\right\}$ estimated over the 30-minute data interval or the standard deviations of the mean values of PSDs at $f=2 f_{\mathrm{gp}}$.
We compute $R_{i}^{j}=g\left(\boldsymbol{X}_{i}^{j}\right)$ and, as already noted, estimate two quantities that characterize resulting error distributions: variance of simulated error distributions, $\sigma_{i}^{2}\left(R_{\mathrm{KAW}}\right)=\sigma^{2}\left(R_{i}^{j}\right)$, and central confidence interval $\left(\mathrm{CI}_{i}\right)$ within which $\approx 68 \%$ simulated values lie, $\left[c i_{\text {lower }}, c i_{\text {upper }}\right]_{i}$. Finally, we introduce a ratio of the upper and lower bounds of CI: $\mathrm{CIF}_{i}=c i_{\text {upper }, \mathrm{i}} / c i_{\text {lower, } \mathrm{i}}$.

\section{ORCID iDs}

Alexander Pitňa (ํ) https://orcid.org/0000-0001-8913-191X Jana Šafránková (i) https://orcid.org/0000-0003-4178-5206

Zdeněk Němeček (i) https://orcid.org/0000-0002-8160-3051

Luca Franci (i) https://orcid.org/0000-0002-7419-0527

Gilbert Pi i https://orcid.org/0000-0002-6039-3622

Victor Montagud Camps (i) https://orcid.org/0000-00027848-9200

\section{References}

Alexandrova, O., Mangeney, A., Maksimovic, M., et al. 2006, JGR, 111, 12208 Alexandrova, O., Saur, J., Lacombe, C., et al. 2009, PhRvL, 103, 165003 Belcher, J. W., \& Davis, L., Jr. 1971, JGR, 76, 3534

Boldyrev, S., Horaites, K., Xia, Q., \& Perez, J. C. 2013, ApJ, 777, 41

Boldyrev, S., \& Perez, J. C. 2012, ApJL, 758, L44

Bruno, R., \& Carbone, V. 2013, LRSP, 10, 2

Cerri, S. S., \& Califano, F. 2017, NJPh, 19, 025007

Chen, C. H. K. 2016, JPIPh, 82, 535820602

Chen, C. H. K., \& Boldyrev, S. 2017, ApJ, 842, 122

Chen, C. H. K., Boldyrev, S., Xia, Q., \& Perez, J. C. 2013a, PhRvL, 110, 225002

Chen, C. H. K., Howes, G. G., Bonnell, J. W., et al. 2013b, in AIP Conf. Ser. 1539, SOLAR WIND 13: Proceedings of the Thirteenth International Solar Wind Conference, ed. G. P. Zank (Melville, NY: AIP), 143

Chen, C. H. K., Mallet, A., Schekochihin, A. A., et al. 2012a, ApJ, 758, 120

Chen, C. H. K., Salem, C. S., Bonnell, J. W., Mozer, F. S., \& Bale, S. D. 2012b, PhRvL, 109, 035001

Chen, C. H. K., Wicks, R. T., Horbury, T. S., \& Schekochihin, A. A. 2010, ApJL, 711, L79

Coleman, P. J., Jr. 1968, ApJ, 153, 371

Cranmer, S. R., Matthaeus, W. H., Breech, B. A., \& Kasper, J. C. 2009, ApJ, 702,1604

Forman, M. A., Wicks, R. T., \& Horbury, T. S. 2011, ApJ, 733, 76

Franci, L., Hellinger, P., Guarrasi, M., et al. 2018a, in J. Phys. Conf. Ser. 1031, 12th Int. Conf. Numerical Modeling of Space Plasma Flows: Astronum2017 (Bristol: IOPP), 012002

Franci, L., Hellinger, P., Matteini, L., Verdini, A., \& Landi, S. 2016, in AIP Conf. Ser. 1720, SOLAR WIND 14: Proc. Fourteenth Int. Solar Wind Conf., ed. L. Wang (Melville, NY: AIP), 040003

Franci, L., Landi, S., Matteini, L., Verdini, A., \& Hellinger, P. 2015, ApJ, 812,21

Franci, L., Landi, S., Verdini, A., Matteini, L., \& Hellinger, P. 2018b, ApJ, 853,26

Galtier, S. 2008, PhRvE, 77, 015302

Goldreich, P., \& Sridhar, S. 1995, ApJ, 438, 763

Grošelj, D., Mallet, A., Loureiro, N. F., \& Jenko, F. 2018, PhRvL, 120, 105101

Hasegawa, A., \& Sato, T. 1989, Space Plasma Physics: I-Stationary Processes (New York: Springer)

Hellinger, P., Trávníček, P. M., Štverák, Š, Matteini, L., \& Velli, M. 2013, JGR, 118, 1351

Hellinger, P., Verdini, A., Landi, S., Franci, L., \& Matteini, L. 2018, ApJL, 857, L19

Hollweg, J. V. 1999, JGR, 104, 14811

Horbury, T. S., Forman, M., \& Oughton, S. 2008, PhRvL, 101, 175005

Horbury, T. S., Wicks, R. T., \& Chen, C. H. K. 2012, SSRv, 172, 325

Howes, G. G., Bale, S. D., Klein, K. G., et al. 2012, ApJL, 753, L19

Howes, G. G., Cowley, S. C., Dorland, W., et al. 2006, ApJ, 651, 590

Howes, G. G., Tenbarge, J. M., Dorland, W., et al. 2011, PhRvL, 107, 035004 Iroshnikov, P. S. 1963, SvA, 40, 742

Klein, K. G., Howes, G. G., TenBarge, J. M., et al. 2012, ApJ, 755, 159

Koval, A., \& Szabo, A. 2013, in AIP Conf. Proc. 1539, SOLAR WIND 13: Proceedings of the Thirteenth International Solar Wind Conference, ed. G. P. Zank (Melville, NY: AIP), 211 
Kraichnan, R. H. 1965, PhFl, 8, 1385

Lacombe, C., Alexandrova, O., \& Matteini, L. 2017, ApJ, 848, 45

Lepping, R. P., Acũna, M. H., Burlaga, L. F., et al. 1995, SSRv, 71, 207

Lion, S., Alexandrova, O., \& Zaslavsky, A. 2016, ApJ, 824, 47

MacBride, B. T., Smith, C. W., \& Forman, M. A. 2008, ApJ, 679, 1644

Matthaeus, W. H., Zank, G. P., Smith, C. W., \& Oughton, S. 1999, PhRvL, 82,3444

Ogilvie, K. W., Chornay, D. J., Fritzenreiter, R. J., et al. 1995, SSRv, 71, 55

Osman, K. T., \& Horbury, T. S. 2007, ApJL, 654, L103

Oughton, S., Matthaeus, W. H., Wan, M., \& Osman, K. T. 2015, RSPTA, 373 , 20140152

Papini, E., Franci, L., Landi, S., et al. 2019, ApJ, 870, 52

Perrone, D., Alexandrova, O., Roberts, O. W., et al. 2017, ApJ, 849, 49

Podesta, J. J. 2009, ApJ, 698, 986

Politano, H., \& Pouquet, A. 1998, PhRvE, 57, R21

Rakhmanova, L., Riazantseva, M., Zastenker, G., \& Verigin, M. 2018, JGR, 123,5300

Riazantseva, M. O., Budaev, V. P., Rakhmanova, L. S., et al. 2016, AdSpR, 58,166

Roberts, O. W., Toledo-Redondo, S., Perrone, D., et al. 2018, GeoRL, 45, 7974
Šafránková, J., Němeček, Z., Němec, F., et al. 2015, ApJ, 803, 107

Šafránková, J., Němeček, Z., Němec, F., et al. 2016, ApJ, 825, 121

Šafránková, J., Němeček, Z., Němec, F., et al. 2019, ApJ, 870, 40

Šafránková, J., Němeček, Z., Přech, L., et al. 2013a, SSRv, 175, 165

Šafránková, J., Němeček, Z., Přech, L., \& Zastenker, G. N. 2013b, PhRvL, 110,025004

Schekochihin, A. A., Cowley, S. C., Dorland, W., et al. 2009, ApJS, 182, 310

Smith, C. W., Vasquez, B. J., \& Hollweg, J. V. 2012, ApJ, 745, 8

Stix, T. H. 1992, Waves in Plasmas (Melville, NY: AIP)

Torrence, C., \& Compo, G. P. 1998, BAMS, 79, 61

Tu, C.-Y., \& Marsch, E. 1995, SSRv, 73, 1

Unti, T. W. J., Neugebauer, M., \& Goldstein, B. E. 1973, ApJ, 180, 591

Verscharen, D., \& Chandran, B. D. G. 2018, RNAAS, 2, 13

Verscharen, D., Chen, C. H. K., \& Wicks, R. T. 2017, ApJ, 840, 106

Wicks, R. T., Horbury, T. S., Chen, C. H. K., \& Schekochihin, A. A. 2010, MNRAS: Letters, 407, L31

Wu, H., Verscharen, D., Wicks, R. T., et al. 2019, ApJ, 870, 106

Zhao, J. S., Voitenko, Y., Yu, M. Y., Lu, J. Y., \& Wu, D. J. 2014, ApJ, 793, 107 